\title{
Intrinsic/Extrinsic: A Relational Account Defended
}

\begin{abstract}
A sentence or statement or proposition that ascribes intrinsic properties to something is entirely about that thing ... A thing has its intrinsic properties in virtue of the way that thing itself, and nothing else, is ... The intrinsic properties of something depend only on that thing ... If something has an intrinsic property, then so does any perfect duplicate of that thing ... (Lewis 1983a, p. 197)
\end{abstract}

I once offered a definition that was meant to capture the notion expressed by the intuitive descriptions in this quote from Lewis. ${ }^{1}$ The basic idea is that $\mathrm{F}$ is an intrinsic property of an item $\mathrm{x}$ just in case x's having $\mathrm{F}$ consists entirely in x's having certain internal properties, where an internal property is one whose instantiation does not consist in one's relation to any distinct items (items other than oneself and one's proper parts). I still think that this relational analysis is largely correct, and here I wish to provide additional support for it and defend it against some objections that have been raised. In the process I aim to make the account somewhat more precise, especially by contrasting it with a grounding approach to defining the intrinsic/extrinsic distinction.

\section{In Favor of a Hyperintensional Account}

Consider those properties that must be exemplified by any item that exists. Following Weatherson and Marshall (2013), let's call them "indiscriminately necessary” properties. Being self-identical is an indiscriminately necessary property. Unlike the property of being identical with Dennett, being self-identical is a property that cannot go unexemplified by anything. In addition to being indiscriminately necessary, the property of being self-identical also seems to be intrinsic; having this property does not seem to be a matter of how an item relates to anything distinct from itself. While this one seems intrinsic, some indiscriminately necessary properties (INPs) appear to be extrinsic. Assuming that numbers exist and exist necessarily, the property of coexisting with the number 9 is an INP. Given realism about numbers, our coexisting with 9 consists in our relation to something distinct from ourselves, which seems to make it an extrinsic feature of you and me.

1 See Francescotti (1999). 
It is well-known that some popular definitions of intrinsicality have the consequence that all INPs are intrinsic. These include the duplication accounts of Lewis (in 1983b and 1986 ${ }^{2}$ ) and Langton and Lewis (1998). ${ }^{3}$ Since the INPs of any item are shared by each of its duplicates, these accounts entail that all INPs are intrinsic. Vallentyne's contraction view also entails that all INPs are intrinsic, since removing from x's world as much as possible while $\mathrm{x}$ remains leaves $x$ with all of its INPs intact. ${ }^{4}$ Since coexisting with 9 (or any other number) certainly seems to be an extrinsic feature of each of us, the consequence that all INPs are intrinsic is a count against these analyses.

However, some have argued that no INPs' being extrinsic is not as implausible as it might seem. Sider mentions that an INP of some object "does not depend in any natural sense on what other objects are like, since this property is necessarily had by every object" (1996, p. 11). Consider, for example, being such that either Socrates is wise or not wise. Despite the reference to Socrates, Sider notes that having this property "doesn't imply any dependence on Socrates, since the property is, of necessity, universally instantiated" (p. 11). HoffmannKolss (2010) mentions that she will exclude INPs from the scope of her discussion, citing the property of being such that $2+3=5$ and claiming that "whether or not an individual $x$ has a non-contingent property of this kind neither depends on what $x$ is like, nor on the environment of $x$, but rather on the logical structure of the property itself" (p. 22). Vallentyne focuses on arguably essential properties of the form having thus-and-such origin (e.g., some specific date or zygote), and contends that it is not so implausible to regard these properties as intrinsic since they are "metaphysically glued" to each of their bearers, and therefore, "in an important sense, there is no dependence since there is no room for variation on what the rest of the world is like" (1997, p. 217). And Harris defends the view that all INPs are intrinsic by appealing to the principle that "[i]f being a concrete object is intrinsic, so, too, is every property that necessarily follows from being a concrete object” (2010, p. 472).

2 See especially 1983b, pp. 355-8 and 1986, pp. 61-3.

3 The account of Langton and Lewis is based on the notion of a property being independent of accompaniment (independent of accompaniment by distinct concrete objects). What they call "basic intrinsic" properties as those that are independent of accompaniment, not disjunctive properties, and not negations of disjunctive properties. They define objects as duplicates just in case they share the same basic intrinsic properties, and then define an intrinsic property as one that can never differ between duplicates.

4 Vallentyne proposes that a property $P$ is intrinsic just in case for any world w, object $x$, and time $t$, if $\mathrm{x}$ is $\mathrm{P}$ in $\mathrm{w}$ at $\mathrm{t}$, then $\mathrm{x}$ is $\mathrm{P}$ at $\mathrm{t}$ in each $\mathrm{x}$-t contraction of $\mathrm{w}$, and likewise for the negation of $P$ (i.e., if $x$ is not-P in $w$ at $t$, it is not-P at each $x$-t contraction of $w$ ). 
These defenses are not entirely convincing. Recall being such that either Socrates is wise or not wise. It seems that this property does depend on the existence of Socrates, for if Socrates did not exist, then neither disjunct would be true, in which case, the disjunction itself would not be true. Our being such that $2+3=5$ also depends on the way the rest of the world is, assuming that numbers exist. Note, also, that these properties qualify as mere Cambridge properties of each of us, ${ }^{5}$ and therefore seem to count as extrinsic in the strongest possible sense. Regarding Vallentyne's point that having a particular origin is metaphysically glued to each of us: rather than proving intrinsicality, the metaphysical glue means that we are essentially related to distinct objects, which suggests not that these features are intrinsic, but rather that some of our essential features are extrinsic. And rather than saying that there is no dependence on what the rest of the world is like since there is no room for variation, it seems more appropriate to say that since there is no room for variation, there is extreme dependence on what the rest of the world is like. ${ }^{6}$ Also, it's not clear why we should agree with Harris that if being a concrete object is intrinsic, then so is every property that necessarily follows from being a concrete object. Suppose that $\mathrm{F}$ is an intrinsic feature of $x$ and that having $F$ necessitates having $G$. Should we then expect that $G$ is an intrinsic feature of $x$ ? We should-only if $x$ 's exemplifying $G$ has solely to do with x's exemplifying F. This is not the case when $\mathrm{G}$ is being accompanied by 9 and $\mathrm{F}$ is being a concrete object. In this case, the former does not have solely to do with the latter since the truth of the former depends on the existence of numbers. In fact, the former seems to have little if anything to do with latter, since for any object $\mathrm{x}$, if numbers exist, then $\mathrm{x}$ is accompanied by 9 whether or not $\mathrm{x}$ is concrete.

As Eddon (2011) reports, "our intuitions about intrinsicality are finegrained," such that "cointensive properties may intuitively diverge with respect to their intrinsicality” (p. 318). That is, our intuitions about intrinsicality seem to entail that

(HI) there are necessarily coextensive properties, F and G, such that $F$ is intrinsic and $\mathrm{G}$ is extrinsic

(where 'HI' stands for 'hyperintensional'). If a property $\mathrm{F}$ of $\mathrm{x}$ is had by all possible duplicates of $\mathrm{x}$, then any property necessarily coextensive with $\mathrm{F}$ is had by all possible duplicates of $\mathrm{x}$. So duplication accounts entail that all INPs are the same in terms of intrinsically (all intrinsic); they therefore entail that $\mathrm{HI}$ is false.

5 I say "each of us" to exclude Socrates himself, and the numbers 2, 3 and 5.

6 I make this point in my 1999, p. 597. 
Since our intuitions about intrinsicality are hyperintensional, as Eddon shows, duplication accounts yield counter-intuitive results in the case of certain INPs.

In addition to $\mathrm{HI}$, there is the related hyperintensionality thesis:

$\left(\mathrm{HI}_{\mathrm{I}}\right) \quad$ there are necessarily coextensive properties, $\mathrm{F}$ and $\mathrm{G}$, such that possibly, there are individuals $\mathrm{x}$ and $\mathrm{y}$, and $\mathrm{x}$ has $\mathrm{F}$ intrinsically while y has G extrinsically.

While $\mathrm{HI}$ mentions properties being intrinsic or extrinsic, $\mathrm{HI}_{\mathrm{L}}$ refers to individuals having properties intrinsically or extrinsically. This distinction between "global" and "local" intrinsicality (as Humberstone calls it") is well-known. Dunn (1990) mentions the difference between "being an intrinsic property (as a kind), and intrinsically being a property of a given individual (as a specific happening)." For example, "if an individual $a$ intrinsically has the property of being square ( $a$ is square by virtue of itself alone), then $a$ also intrinsically has the property of being square-or-accompanied ( $a$ is square-or-accompanied by virtue of itself alone)" (p. 183); however, being square-or-accompanied is had extrinsically by an accompanied circular object. So, we see, the same property can be had intrinsicality by one item and extrinsically by another. Humberstone (1996, p. 228) gives the example of being either made of tin or adjacent to something made of tin, a property which is exemplified intrinsically by tin objects but extrinsically by nearby tinless items.

Some INPs illustrate $\mathrm{HI}_{\mathrm{L}}$. Being accompanied by the number 9 is an extrinsic property of each of us, but it seems to be intrinsic to the number 9 itself. The non-INPs mentioned by Dunn and Humberstone also qualify as instances of $\mathrm{HI}_{\mathrm{L}}$. Since any property is necessarily coextensive with itself, any time a property can be instantiated both intrinsically and extrinsically, there is an F that can be instantiated intrinsically and a necessarily coextensive $\mathrm{G}$ (which is $\mathrm{F}$ ) that can be instantiated extrinsically. So the mixed-cases mentioned in the previous paragraph seem to establish the truth of $\mathrm{HI}_{\mathrm{L}}$. And as Figdor (2008) reveals, these mixed cases include more than just philosophers' designer properties. Many less contrived properties can be used to support $\mathrm{HI}_{\mathrm{L}}$. Figdor has us consider the property of being witty, which Falstaff has in and of himself, and which Prince Hal has extrinsically since he is witty only in the presence of other witty people. She also mentions, among other examples, the property of being valuable, which might be exemplified by some things in virtue of what they are like in themselves, and by other things in virtue of their relational features (e.g., their consequences). In fact, if one item can be valuable intrinsically

7 See Humberstone (1996, pp. 227-8). 
while another item is valuable extrinsically, then as Figdor $(2014)^{8}$ describes, it is also possible that the same item has value intrinsically (due to its internal properties) and extrinsically (e.g., because it is valued by others) at the very same time. This is an uncontrived, ultra-mixed case-the same property had by the same individual at the same time both intrinsically and extrinsically. ${ }^{9}$ In his recent defense of the hyperintensionality of intrinsicality, Bader (2013) also mentions cases in which a property is possessed by an individual both intrinsically and extrinsically. ${ }^{10}$

Given the plausibility of $\mathrm{HI}$ and $\mathrm{HI}_{\mathrm{L}}$, one might like to know which account of intrinsicality best accommodates both. ${ }^{11}$ One natural way to honor both is with an appeal to the notion of grounding. The grounding approach is discussed in the following sections, along with the non-grounding view that I favor.

\section{The Grounding Approach}

Talk of grounding is meant to capture the intuitive idea of one fact consisting in or holding in virtue of another fact. Examples of grounding talk include the claim that moral facts obtain in virtue of non-moral facts, that facts about mentality consist in nothing more than physical facts, and that dispositional facts are grounded in categorical properties. ${ }^{12}$

8 See Figdor's paper, "What's the Value of an Intrinsic Property?", which she wrote for this collection.

9 The possibility of these ultra-mixed cases is suggested by Figdor's 2008 discussion. Talk of having properties intrinsically, she claims, indicates independence from contextually relevant counterfactual circumstances, and which counterfactual circumstances are relevant and when depends on our explanatory purposes, as she describes in detail. If this explanatory account is correct, then there is reason to suspect that depending on our explanatory purposes, property $\mathrm{F}$ might count as one that $\mathrm{x}$ has intrinsically at $\mathrm{t}$ or as one that $\mathrm{x}$ has extrinsically at $\mathrm{t}$.

10 The mixed cases make it tempting to regard the local notion of intrinsicality as more fundamental than the global notion, as Figdor $(2008,2014)$ and Bader (2013) make clear. Although see Witmer (2014), in this anthology, for an intriguing defense of the claim that the global notion is in fact the more basic.

11 One might argue that despite the evidence to the contrary (offered by Eddon, Figdor, and Bader), a hyperintensional approach should actually be rejected. See Hoffmann-Kolss' "Is the Intrinsic/Extrinsic Distinction Hyperintensional?," in this collection, for a strong defense of the view that the notion of intrinsicality is not hyperintensional. I do not deny that a decent case can be made against the hyperintensionality claim. However, what I wish to focus on here is what definition of 'intrinsic' we should accept assuming that we do wish to honor $\mathrm{HI}$ and $\mathrm{HI}_{\mathrm{L}}$.

12 For a list that includes these and other examples of grounding talk, see Correia (2010, p. 251). 
Many who defend the notion of grounding as a valuable philosophical concept have tried to explain what they consider some of its most distinctive logical features in an effort to make the notion less than unacceptably obscure. ${ }^{13}$ One feature often considered crucial to grounding is necessitation; it is claimed that one fact is grounded in another only if the latter necessitates the former. In general, "If [p] is grounded in [q], then q entails p," and when grounding is understood as a type of metaphysical dependence, the "facts that ground [p] together ensure as a matter of metaphysical necessity that [p] obtains" (Rosen 2010, p. 118). Another logical feature commonly cited in discussions of grounding is asymmetry. It is thought that if one set of facts is grounded in another, then the latter is not grounded in the former. The appeal to asymmetry honors the idea that the ground is more fundamental than the facts that are grounded. Because the ground is thought of as more fundamental, irreflexivity has also been considered definitive of grounding since no fact can be more fundamental than itself. Transitivity is also mentioned in discussions of grounding since it is tempting to think that if (for example) a moral fact is grounded in a behavioral fact and the behavioral fact is grounded in a microphysical fact, then the moral fact is grounded in the microphysical fact. ${ }^{14}$

To illustrate the potential usefulness of the notion of grounding, Rosen gives examples of how the notion might help solve various metaphysical issues, including its potential use in analyzing intrinsicality. On "one intuitive gloss," Rosen notes, "a property $\mathrm{F}$ is intrinsic iff whether or not X is F depends entirely on how things stand with $\mathrm{X}$ and its parts, and not on X's relations to things distinct from X" (2010, p. 112). This intuitive characterization of intrinsicality, Rosen claims, can be made explicit as follows:

$\mathrm{F}$ is an intrinsic property iff, as a matter of necessity, for all $\mathrm{x}$ :

If $\mathrm{x}$ is $\mathrm{F}$ in virtue of $\phi(\mathrm{y})$-where $\phi(\mathrm{y})$ is a fact containing $\mathrm{y}$ as a constituentthen $\mathrm{y}$ is part of $\mathrm{x}$; and

If $x$ is not-F in virtue of $\phi(y)$, then $y$ is part of $x .(p .112)$

Like grounding accounts generally, this one is hyperintensional. ${ }^{15}$ Being accompanied by the number 9 is grounded in the fact that 9 exists, and this fact has the number 9 as a constituent, a constituent that obviously is not a part of you or me. So being accompanied by 9 is plausibly classified as one of our extrinsic

13 Rosen (2010) provides a helpful description of the logical and structural features characteristic of grounding.

14 Although, Schaffer (2012) offers counter-examples to the transitivity of grounding.

15 I say "like grounding accounts generally" since the notion of grounding itself is hyperintensional. 
properties. On the other hand, being self-identical is classified as intrinsic, since for any $\mathrm{x}$, x's being self-identical is grounded in the fact that $\mathrm{x}=\mathrm{x}$, and $\mathrm{x}$ trivially is a part of $x$. Both features are INPs, but on Rosen's account only one of them is intrinsic.

When applied locally, Rosen's account also correctly classifies non-INP mixed cases. Being accompanied by Fido is an extrinsic property of us but it's exemplified intrinsically by Fido since our being accompanied by Fido has a constituent that is distinct from us, but Fido's being accompanied by Fido does not have any constituent distinct from Fido. Being either made of tin or adjacent to something made of tin is an intrinsic feature of tin object $\mathrm{x}$ on the local version of Rosen's account since $x$ has this property in virtue of the fact that it is made of tin and this fact does not contain any object that is not part of $\mathrm{x}$. But any tinless object that has the disjunctive property has it extrinsically since it has it in virtue of the fact that it is adjacent to something made of tin, a fact which contains something that is not part of the tinless object.

By honoring $\mathrm{HI}$ and $\mathrm{HI}_{\mathrm{L}}$, Rosen's definition yields the intuitively correct result in these cases and each of the others mentioned earlier. Another version of the grounding approach that provides the same plausible results is the grounding definition Witmer develops in "A Simple Theory of Intrinsicality," written for this collection. On this view, $F$ is an intrinsic property of $x$ just in case its presence or absence is grounded only in facts that are "parts" of $\mathrm{x}$ (where the notion of x's spatial parts is replaced by Witmer with the notion of that on which $\mathrm{x}$ is ontologically dependent). ${ }^{16}$

16 Witmer, Butchard, and Trogdon (2005) offer a grounding account that retains the idea of Langton and Lewis that intrinsic properties are independent of accompaniment. They propose: "Property $\mathrm{P}$ is intrinsic iff, for any possible individual $\mathrm{x}$, if $\mathrm{x}$ has $\mathrm{P}, \mathrm{x}$ has $\mathrm{P}$ in an intrinsic fashion," where " $\mathrm{x}$ has $\mathrm{P}$ in an intrinsic fashion iff (i) $\mathrm{P}$ is independent of accompaniment and (ii) for any property $\mathrm{Q}$, if $\mathrm{x}$ has $\mathrm{P}$ in virtue of having $\mathrm{Q}, \mathrm{Q}$ is also independent of accompaniment" (p. 333). With the phrase 'in virtue of' they seem to be utilizing the notion of grounding, for they claim that with their account "we are directed to look at the bottom-level properties that ground the others" (p. 340; their emphasis). And with the appeal to grounding, their definition improves on the Langton-Lewis account at least by allowing that in some cases "two properties which are necessarily coextensive can yet differ in whether they are intrinsic” (p. 334). However, although the account is hyperintensional, by requiring independence from accompaniment as a necessary condition for intrinsicality, the account is arguably not hyperintensional enough, and in particular, it does not classify any INP as intrinsic. (They defend this consequence by noting that while an INP does not depend on anything outside the individual, "it doesn't depend on anything inside the individual either-it is simply independent of everything” (p. 348). However, for any $\mathrm{x}$, being self-identical does seem to depend, and depend entirely, on $\mathrm{x}$ itself.) 
However, there are other ways to accommodate $\mathrm{HI}$ and $\mathrm{HI}_{\mathrm{L}}$, ways that do not rely on the notion of grounding. Late in the next section, I mention one such way.

\section{A Relational Approach}

On a relational account of the intrinsic/extrinsic distinction,

(R) whether F is extrinsic or intrinsic (or had extrinsically or intrinsically) is a matter of whether having $\mathrm{F}$ depends on relations to distinct items. ${ }^{17}$

One possible way to understand the phrase 'depends on' in $\mathrm{R}$ is in terms of the necessary biconditional. On this view F is extrinsic (or had extrinsically) just in case there is a relation such that, necessarily, an object has $\mathrm{F}$ if and only if it bears that relation to a distinct thing, and otherwise $\mathrm{F}$ is intrinsic (had intrinsically). However, biconditionals, even necessary biconditionals, are not hyperintensional. So if we want a relational account that's compatible with $\mathrm{HI}$ and $\mathrm{HI}_{\mathrm{L}}$, we need to rely on something other than biconditionality. ${ }^{18}$

We might understand the dependence relation mentioned in $\mathrm{R}$ in terms of grounding, and then we would have a grounding account that is also relational. It seems that Rosen's proposal is exactly of this sort; recall his intuitive characterization- "a property $\mathrm{F}$ is intrinsic iff whether or not X is F depends entirely on how things stand with $\mathrm{X}$ and its parts, and not on X's relations to things distinct from X” (2010, p. 112). A grounding-relational account of the intrinsic/extrinsic distinction is any account that takes the form:

$\left(\mathrm{R}_{\mathrm{G}}\right) \quad$ whether $\mathrm{F}$ is extrinsic or intrinsic (or had extrinsically or intrinsically) is a matter of whether having $\mathrm{F}$ is grounded in relations to distinct items. ${ }^{19}$

Instead of relying on the notion of grounding, one can honor $\mathrm{HI}$ and $\mathrm{HI}_{\mathrm{L}}$ by appealing to identity, with a relational view of the form:

17 Again, $\mathrm{y}$ is distinct from $\mathrm{x}$ iff $\mathrm{y}$ is not identical with $\mathrm{x}$ or any of $\mathrm{x}$ 's proper parts.

18 Hoffmann-Kolss' (2010) relational account, like my own, is based on the idea that one's intrinsic properties are those that do not consist in one's relations to distinct objects. But by construing the notion of consisting in at work here in terms of necessary biconditionals, her account entails that $(\mathrm{HI})$ and $\left(\mathrm{HI}_{\mathrm{L}}\right)$ are false.

19 A grounding approach does not need to rely on the notion of relationality; e. g., the grounding account of Witmer, Butchard, and Trogdon (2005) relies, instead, on the notion of being independent of accompaniment. 
$\left(\mathrm{R}_{\mathrm{I}}\right) \quad$ whether $\mathrm{F}$ is extrinsic or intrinsic (or had extrinsically or intrinsically) is a matter of whether having $\mathrm{F}$ is (identical with, the very same as) being suitably related to distinct items.

My 1999 account is an instance of R. The proposal was that

$\left(\mathrm{R}^{\star}\right) \quad \mathrm{F}$ is an intrinsic property of item $\mathrm{x}={ }_{\mathrm{df}} \mathrm{x}$ has $\mathrm{F}$, and there are internal properties $I_{1}, \ldots, I_{n}$ had by $x$, such that x's having F consists in x's having $\mathrm{I}_{1}, \ldots, \mathrm{I}_{\mathrm{n}}$,

where an internal property is one that is not d-relational, and where d-relations are relations one bears to distinct items. ${ }^{20}$ The "consists in" relation mentioned in $\mathrm{R}^{\star}$ could be understood in terms of grounding, and in that case the definition would be an instance of $\mathrm{R}_{\mathrm{G}}$. But I chose, instead, to view the "consists in" relation, in this context, as identity. I claimed that for the purpose of $\mathrm{R}^{\star}$, "the event or state, x's having F, consists in the event or state, x's having G, just in case $x$ 's having $F$ is the very same event or state as x's having G" (p. 599). The result is

$\left(\mathrm{R}_{\mathrm{I}}^{\star}\right) \quad \mathrm{F}$ is an intrinsic property of item $\mathrm{x}={ }_{\mathrm{df}} \mathrm{x}$ has $\mathrm{F}$, and there are internal (non-d-relational) properties $\mathrm{I}_{1}, \ldots ., \mathrm{I}_{\mathrm{n}}$ had by $\mathrm{x}$, such that x's having $\mathrm{F}$ is the same as x's having $\mathrm{I}_{1}, \ldots, \mathrm{I}_{\mathrm{n}}{ }^{21}$

According to $\mathrm{R}_{\mathrm{I}}{ }^{\star}$, being self-identical is an intrinsic property of $\mathrm{x}$ since there is an internal, non-d-relational property, I, where I is being identical with $x$ and $\mathrm{x}$ 's being self-identical is same event or state as x's being identical with $\mathrm{x}$. The necessarily coextensive property, being accompanied by the number 9, is classified as an extrinsic property of you and me since our having this property is nothing more or less than our bearing the right relation (the coexisting relation)

20 Borrowing from Khamara's (1988) characterization of different types of relational properties, I mentioned different types of d-relational properties. $\mathrm{F}$ is a positive, existential, impure drelational property of an item $x$ just in case there is a relation $R$, and an item $y$, such that x's having $\mathrm{F}$ consists in $\mathrm{x}$ 's bearing $\mathrm{R}$ to $\mathrm{y}$, and $\mathrm{y}$ is distinct from $\mathrm{x}$. $\mathrm{F}$ is a positive, existential, pure $\mathrm{d}$ relational property of $\mathrm{x}$ just in case there is a relation $\mathrm{R}$, and a class of items $\mathrm{C}$, such that x's having $\mathrm{F}$ consists in there being some member of $\mathrm{C}$ to which $\mathrm{x}$ bears $\mathrm{R}$, and at least one member of $C$ to which $x$ bears $R$ is distinct from $x$. And as Humberstone reminds us (1996, p. 212), an existential relational property has a universal counterpart. A positive universal d-relational property of $\mathrm{x}$ is such that there is a relation $\mathrm{R}$, and a class of items $\mathrm{C}$, such that $\mathrm{x}$ 's having $\mathrm{F}$ consists in x's bearing $\mathrm{R}$ to every member of $\mathrm{C}$, and there is a possible member of $\mathrm{C}$ that is distinct from $\mathrm{x}$. There are also the negative counterparts of each of these positive d-relational properties. And variations on any of these can be used to describe other types of d-relationality; e.g., with relations that are more than two-place.

21 The identity construal is also meant to apply to the phrase 'consists in' that figures in the descriptions of the various d-relational properties. 
to a distinct item (the number 9). Thus, with $\mathrm{R}_{\mathrm{I}}{ }^{\star}$ we allow the truth of $\mathrm{HI}$. $\mathrm{R}_{\mathrm{I}}{ }^{\star}$ also allows mixed cases where the same property can be had intrinsically and also extrinsically, which entails $\mathrm{HI}_{\mathrm{L}}$. Being either square or accompanied is an intrinsic feature of the lonely square, according to $\mathrm{R}_{\mathrm{I}}{ }^{\star}$, because the lonely square's having the disjunctive property is nothing other than its being square, which is an internal (non-d-relational) property. However, the accompanied circle has the property extrinsically since its being square or accompanied is simply its being accompanied, which is d-relational. The ultra-mixed cases described by Figdor (2008, 2014) are also allowed by $R_{I}{ }^{\star}$. It might be that at the very same time $x$ has value intrinsically and also extrinsically-where there is some internal property I and some d-relational property D, such that x's intrinsic instantiation of value is its having I, and x's extrinsic instantiation of value is its having D.

$\mathrm{R}_{\mathrm{I}}$ * is compatible with $\mathrm{HI}$ and $\mathrm{HI}_{\mathrm{L}}$. But so is a grounding approach (including grounding views of the relational variety, $\mathrm{R}_{\mathrm{G}}$ ). So for those who wish to honor $\mathrm{HI}$ and $\mathrm{HI}_{\mathrm{L}}$, the question is: which is preferable $-\mathrm{R}_{\mathrm{I}}{ }^{\star}$ or an account of intrinsicality that appeals to grounding? Maybe the correct answer is "neither": maybe some altogether different approach is the best way to hyperintensionally define the intrinsic/extrinsic distinction. However, here I focus on the choice between $R_{I}{ }^{\star}$ and a grounding account.

\section{Grounding or Identity?}

Recall Rosen's proposal that $\mathrm{F}$ is an intrinsic property just in case, necessarily, for any $x$, (i) if $x$ is F in virtue of $\phi(y)$, then $y$ is part of $x$; and (ii) if $x$ is not- $F$ in virtue of $\phi(y)$, then $y$ is part of $x$. In his discussion of this view, Marshall (2013, sec. 2) mentions a worry about fundamental properties. Given that no fact can ground itself, if a fact is fundamental, then it is not grounded. By the phrase, 'in virtue of', Rosen has the grounding relation in mind. So for any $x$ and F such that x's having $F$ is a fundamental fact and x's lacking $F$ is also fundamental, the two conditionals, (i) and (ii), in Rosen's definition are vacuously true. Thus, on Rosen's account, any property whose presence or absence is a fundamental fact is intrinsic. However, we might wish to allow extrinsic properties whose presence or absence is fundamental; at least, a definition of 'intrinsic' should not rule out this possibility.

We can avoid the result that all properties whose presence or absence is fundamental are intrinsic properties by adding, prior to (i) and (ii), the condition that if $\mathrm{x}$ is $\mathrm{F}$ (or not-F), then x's being F (or not-F) is grounded, i.e., that there is some fact $\phi(y)$ in virtue of which $x$ is $F$ (or not-F). Of course, then we get the even more implausible result that whenever either $\mathrm{x}$ is $\mathrm{F}$ or $\mathrm{x}$ is not-F is fun- 
damental, $\mathrm{F}$ is not an intrinsic property. A major worry, then, for grounding accounts is how to express the grounding intuition in a way that yields plausible results in the case of fundamental facts. ${ }^{22}$

$\mathrm{R}_{\mathrm{I}}{ }^{\star}$, on the other hand, has no problem correctly classifying fundamental facts about the presence or absence of intrinsic properties. Suppose that $\mathrm{x}$ contains $\mathrm{y}$ as a proper part, and that this is a wholly fundamental fact, not grounded in anything more basic. Then according to $\mathrm{R}_{\mathrm{I}}{ }^{\star}$ containing $y$ as a proper part is an intrinsic feature of $\mathrm{x}$, despite its fundamentality, since containing $y$ as a proper part is an internal (non-d-relational) feature of $\mathrm{x} . \mathrm{R}_{\mathrm{I}}{ }^{\star}$ also allows that some fundamental facts are facts about the presence or absence of extrinsic properties. Suppose it is a primitive fact that atom $\mathrm{x}$ is to the left of atom $\mathrm{y} \cdot \mathrm{R}^{\star}{ }_{1}$ correctly classifies being to the left of $y$ as extrinsic despite its fundamentality since $x$ 's having this property is its bearing the left of relation to the distinct object $\mathrm{y}$.

Marshall mentions that a grounding theorist might try to avoid the result that all fundamental properties are intrinsic with the following revision of Rosen's proposal:

$\mathrm{F}$ is an intrinsic property just in case, necessarily, for any $\mathrm{x}$, (i) if $\mathrm{x}$ is $\mathrm{F}$ in virtue of, or is identical with, $\phi(\mathrm{y})$, then $\mathrm{y}$ is part of $\mathrm{x}$; and (ii) if $\mathrm{x}$ is not-F in virtue of, or is identical with, $\phi(\mathrm{y})$, then $\mathrm{y}$ is part of $\mathrm{x} .{ }^{23}$

With this account, fundamental facts of the form ' $\mathrm{x}$ is F' and ' $\mathrm{x}$ is not-F' are not prevented from being extrinsic. Suppose that for some $x$, $x$ 's being $F$ (not-F) is identical with $\phi(\mathrm{y})$. If $\mathrm{y}$ is distinct from $\mathrm{x}$, then $\mathrm{F}$ (not-F) is extrinsic, according to $\mathrm{G}^{\star}$, even if $\mathrm{x}$ 's being $\mathrm{F}$ (not-F) is fundamental and therefore ungrounded.

Yet, Marshall (2013, sec. 3) notes, there is another major objection to a grounding analysis of intrinsicality, one which threatens the revision as well as Rosen's original. It seems possible for x's having some intrinsic property to be grounded in facts about items that are not parts of $\mathrm{x}$. Marshall has us consider the singleton set, \{Obama\}. The fact that \{Obama\} exists is grounded in the fact that Obama exists. But Obama himself is not literally a part of any set since sets are abstract objects. It would seem to follow that \{Obama\}'s existence is ground-

22 The account of Witmer, Trogdon, and Butchard (2005) allows that some fundamental properties are intrinsic and some are not. Yet, despite their appeal to grounding, the independence from accompaniment component gives the implausible result that all INPs are extrinsic (as mentioned in note 16).

23 This is my phrasing, not Marshall's. Incidently, Marshall points out that this account like the original can be reformulated so as not to presuppose a Russellian view of facts (a view on which facts are structured entities with individuals and properties as constituents). As Marshall notes, the account can be rephrased to mention facts concerning rather than containing an individual y. 
ed in a fact concerning that which is not a part of \{Obama\}. Marshall concludes that on Rosen's account, existing ends up being an extrinsic property of \{Obama\}, clearly an undesirable result since existence would seem to be intrinsic to all things that exist.

One might argue that this case is not a threat to Rosen's proposal or the revised version since the notion of parthood utilized in a definition of intrinsicality can be, and should be, liberal enough to include more than just spatial parts. Temporal segments should also be included so that, for example, the property of containing many minutes may be considered an intrinsic property of an hour; and the temporal parts that perdurantists speak of certainly count as genuine parts, assuming they exist. Set membership should arguably also be considered a type of parthood. And if so, then the existence of \{Obama\} being grounded in the existence of Obama need not be considered a threat. ${ }^{24}$

Still, it seems there are possible cases in which facts about the intrinsic properties of an individual are grounded in facts about things that are not among what plausibly count as it parts. One might believe, for example, that the totality of contingent beings depends for its existence on some necessary being, where the dependence is such that the existence of the latter grounds the existence of the former. While this belief may be false, it is a belief one can consistently endorse while also holding that contingent beings have intrinsic properties. Suppose, for example, that an object $\mathrm{x}$ is comprised of gold. Being comprised of gold would seem to be one of x's intrinsic properties even if $\mathrm{x}$ would not have been made of gold and would not have even existed if not for the presence of some distinct being. If one were to acquire the belief that there is a supreme being on which all contingencies ultimately depend (including the existence of the gold object and its having the properties it has), it seems one wouldn't thereby need to deny that some of its properties are intrinsic. So it seems possible for the instantiation of an intrinsic property to depend in some intimate way, and a way that might be labeled "grounding," on facts about distinct items. And if so, then a definition of intrinsicality should not prevent intrinsic properties from ultimately being grounded in facts about distinct items. ${ }^{25}$

24 Marshall mentions that one can employ a broadened notion of a part (the notion of a "generalised part"), which includes the membership relation. However, he worries that for the definition to remain compatible with a variety of different metaphysical theories, there is no telling how much the notion of a generalized part will need to include, making a satisfactory version of the grounding account difficult if not impossible to achieve.

25 Trogdon (2009) illustrates this point with the help of priority monism. On this view, facts about the world as a whole ground the facts about the world's proper parts. While many may find this view implausible, the worry remains: if priority monism were true, then on a grounding 
Perhaps a plausible grounding analysis can be developed so as to allow properties to be intrinsic despite their grounding in facts about distinct items; although, this remains to be seen. Note, however, that $R_{I}{ }^{\star}$ easily allows intrinsic properties to depend in some intimate way, and a way that might be called "grounding," on relations to distinct items. According to $\mathrm{R}_{\mathrm{I}}{ }^{\star}$, existing is an intrinsic property of singleton \{Obama\} simply because there is an internal (non-d-relational) property I, where I is the property of existing, and \{Obama\}'s existing is the very same as $\{\mathrm{Ob}$ ama\}'s having I. Granted, \{Obama\}'s existing depends in some significant sense on Obama existing, but \{Obama\}'s existing is not the same state of affairs as Obama's existing. \{Obama\}'s existing is its possession of an internal property, not its possession of the apparently d-relational property of being such that Obama exists. So, with $\mathrm{R}_{\mathrm{I}}{ }^{\star}$, even assuming that Obama is not considered part of \{Obama\}, existing counts as an intrinsic property of \{Obama\}. Or suppose there is a god, and this god is the ultimate source of all contingencies-that on which all contingencies depend. Being comprised of gold would still be an intrinsic feature of object $\mathrm{x}$, according to $\mathrm{R}_{\mathrm{I}}{ }^{\star}$, since $\mathrm{x}$ 's having this property is its having the internal property of containing large amounts of $\mathrm{Au}$. Whether there is a distinct being on which x's containing large amounts of Au depends does not change the fact that being comprised of gold (containing large amounts of $\mathrm{Au}$ ) is not itself a d-relational feature. It seems, then, that $R_{I}{ }^{*}$ can allow that $F$ is intrinsic to $x$ even if $x$ 's being $F$ is grounded in facts about how $\mathrm{x}$ relates to distinct things.

There is another reason to prefer $\mathrm{R}_{\mathrm{I}}{ }^{\star}$ to a grounding view. The notion of an intrinsic property does not itself imply anything about whether the intuitive notion of grounding, as described in the literature, is a relation that actually ob-

account of intrinsicality, none of the properties of any proper parts of the world would be intrinsic, for each property would be instantiated by the parts in virtue of their relation to the whole world.

To make priority monism consistent with a grounding view, Trogdon (2009) modifies the original Witmer, Butchard and Trogdon (2005) account by distinguishing between intra- and inter-level grounding (being instantiated intra-virtue-of vs. inter-virtue-of). He proposes that " $\mathrm{x}$ has $\mathrm{P}$ in an intrinsic fashion just in case (i) $\mathrm{P}$ is independent of accompaniment; and (ii) for any individual $y$ and property $\mathrm{Q}$, if $\mathrm{x}$ has $\mathrm{P}$ intra-virtue-of y's having $\mathrm{Q}$, then $\mathrm{Q}$ is either fundamental or independent of accompaniment” (2009, p. 143). Since properties of the proper parts of the world are had inter-virtue-of properties of the whole, they are not precluded from being intrinsic with this new view. There is, however, the worry that by specifying only intra-level grounding in condition (ii), the revised definition gives the result that all properties that are inter-level grounded are classified as intrinsic so long as they are independent of accompaniment. Skiles (2009) describes an extrinsic property that seems to satisfy (i) and (ii) by being independent of accompaniment and grounded in relations that cross mereological levels. However, see Trogdon's (2010) reply to Skiles' objection. 
tains. It is a matter of controversy whether talk of grounding should be embraced or viewed with suspicion, and it is a matter of controversy even for those who take the intrinsic/extrinsic distinction seriously. It seems, then, that a definition of intrinsicality should remain neutral on whether grounding is actually a real phenomenon. To its credit, $R_{I}^{\star}$ does remain neutral on this issue. $R_{1}^{\star}$ tells us that what makes $\mathrm{F}$ an intrinsic property of $\mathrm{x}$ is that there is an internal, nond-relational property (or set of such properties) I, such that x's having F is x's having I. That there is this identity leaves it completely open whether x's having F, i.e., x's having I, is itself grounded in some more fundamental fact, or whether talk of grounding ever describes anything that actually obtains.

So there are some reasons to prefer $\mathrm{R}_{\mathrm{I}}{ }^{\star}$ to a grounding account. I wish to provide further support for $\mathrm{R}_{\mathrm{I}}{ }^{\star}$ by answering some objections to the definition that have been raised.

\section{Objections to $R_{1}^{*}$}

a. Weatherson and Marshall (2013, sec. 2.1) present a general worry for relational accounts of intrinsicality, a worry that applies to $R_{I}{ }^{\star}$ in particular. They mention the property, not being within a mile of a rhododendron, and note that a non-rhododendron all alone in the world can have this property without its bearing a relation to any non-part. Since the lonely non-rhododendron's having this property does not consist in any relation to a non-part, the worry is that a relational account yields the implausible result that the lonely non-rhododendron has the property intrinsically.

On some possible relational accounts, for $\mathrm{F}$ to be an extrinsic feature of $\mathrm{x}$, there must exist a distinct item y such that x's having $\mathrm{F}$ consists in x's bearing the right relation or relations to $y$. But a relational account need not, and should not, have this requirement. A property that consists in a relation one bears to a distinct item qualifies as extrinsic since it is a property that one has in virtue of what the rest of the world is like. The negation of such a property requires that there is no distinct item to which one is related in that way. So the negation is also a matter of what the rest of the world is like, and therefore should also be considered extrinsic. It seems, then, that what a relational account should entail is that if $\mathrm{F}$ is an extrinsic feature of $\mathrm{x}$, then x's having $\mathrm{F}$ consists in either $\mathrm{x}$ 's bearing a certain relation to a distinct thing or x's not bearing that relation to a distinct thing. And a relational account will entail this disjunction so long as the relations to distinct items that make for extrinsicality are taken to include negative as well as positive d-relations. Being within a mile of a rhododendron is a positive (existential, pure) d-relational property of $\mathrm{x}$; to be within a mile of a rho- 
dodendron is for there to be a member of the class of rhododendrons which is distinct from $\mathrm{x}$ and to which $\mathrm{x}$ bears the within a mile of relation. The negation of this positive d-relational property is not being within a mile of a rhododendron, and x's having this negative d-relational property consists in there being no member of the class of rhododendrons to which $\mathrm{x}$ bears the within a mile of relation. The lonely non-rhododendron has this negative d-relational property. So by including negative d-relations in the class of d-relations that yield extrinsicality, proponents of a relational account can ensure that not being within a mile of a rhododendron counts as an extrinsic feature of the lonely non-rhododendron.

b. Weatherson and Marshall (2013, sec. 3.5) claim that my definition is incompatible with certain accounts of property-identity. Consider, for example, a coarsegrained view according to which, (i) all necessarily co-extensive properties are identical. Each property is necessarily co-extensive with a d-relational property; e.g., any property $\mathrm{F}$ is necessarily co-extensive with being either ( $F$ and lonely) or ( $F$ and accompanied). So given (i), each property is identical with a d-relational property. Add $\mathrm{R}_{\mathrm{I}}{ }^{\star}$ and we get the result that all properties are had extrinsically. But, surely, a definition of 'intrinsic' should not itself prevent properties being had intrinsically. So the objection is: if $\mathrm{R}_{\mathrm{I}}{ }^{\star}$ is to allow that some properties are sometimes had intrinsically, as it should, then it cannot allow the truth of (i), which is a problem since a definition of 'intrinsic' should remain neutral on how finely or coarsely properties are to be individuated.

Granted, a definition of 'intrinsic' should remain neutral on how finely or coarsely we should individuate properties. However, $\mathrm{R}_{\mathrm{I}}{ }^{\star}$ is perfectly compatible with (i). We can consistently accept $\mathrm{R}_{\mathrm{I}}{ }^{\star}$ along with (i) simply by allowing that no properties are ever had intrinsically. This consequence may be considered implausible, but it's not clear that whatever implausibility it has is any threat to $\mathrm{R}_{\mathrm{I}}{ }^{\star}$. For, as Weatherson and Marshall concede, if we wish to avoid the result that there is no intrinsicality, then rather than rejecting $\mathrm{R}_{\mathrm{I}}{ }^{\star}$, we can easily reject the coarse-grained view of property-identity instead. And in favor of this latter option note that with any definition of intrinsicality, (i) yields the implausible view that every $\mathrm{F}$ is in every case extrinsic (given that the necessarily coextensive being either $F$ and lonely or F and accompanied is always had extrinsically). So, in response to Weatherson and Marshall, there are two points to stress: $R_{1}{ }^{\star}$ is neutral regarding (i) since $R_{I}{ }^{\star}$ is consistent with the view that no properties are had intrinsically, and while this consequence may be implausible, its implausibility arguably counts more against (i) than against $\mathrm{R}_{1}{ }^{26}$

26 Weatherson and Marshall (2013, sec. 3.5) contend that my definition is also incompatible 
c. For the purpose of my definition, I understood the 'consists in' that figures in $\mathrm{R}^{\star}$ above in terms of identity. The proposal was that "the event or state, $\mathrm{x}$ 's having $\mathrm{F}$, consists in the event or state, x's having G, just in case $x$ 's having $F$ is the very same event or state as X's having G” (1999, p. 599). Hoffmann-Kolss (2010, pp. 91-2) worries that with the absence of detail about what makes events or states identical, my definition, $\mathrm{R}_{\mathrm{I}}{ }^{\star}$, does not sufficiently elucidate the notion of intrinsicality, and for all we can tell from the scant details provided, the definition might very well give implausible results. As Hoffmann-Kolss indicates, for all I say about event- or state-identity, my definition allows, for example, that for any arbitrary chosen property $\mathrm{F}$ and any object $\mathrm{x}$,

(iii) $\quad x$ 's having $F$ is the same event/state as $x$ 's having $F$ and having the same pure properties as every qualitatively identical $y^{27}$

However, it would seem that if (iii) is true, then all properties are d-relational (since having the same pure properties as every qualitatively identical $y$ is a universal d-relational property). So if (iii) is true, then there is no internal property I such that X's has F is X's having I. So by adding (iii) to $\mathrm{R}_{\mathrm{I}}{ }^{\star}$ we get the result that no properties are intrinsic or had intrinsically. The worry, then, is that in the absence of details about event/state-identity, details which might entail that (iii) is false, $\mathrm{R}_{\mathrm{I}}{ }^{\star}$ threatens to preclude all intrinsicality.

with certain fine-grained accounts of property-identity. Consider the view that (ii) properties are structured entities whose structure matches the syntactic structure of the predicates that express them. Given (ii), the property of being accompanied is not identical with the d-relational property of being such that there is a contingently existing $x$ which one is distinct from and co-exists with given the difference in syntactic structure, and it seems that if (ii) were true, then being accompanied would not be identical with any d-relational property. So the worry is that with $\mathrm{R}_{\mathrm{I}}{ }^{*}$, being accompanied is falsely classified as intrinsic. It would seem, then, that to give the right results, $\mathrm{R}_{\mathrm{I}}^{\star}$ cannot allow the truth of (ii).

Granted, an account of intrinsicality, relational or otherwise, should not force us to reject (ii). And $R_{I}{ }^{\star}$ does not force us to reject it. In fact, we can easily endorse (ii) along with $R_{I}{ }^{\star}$, and all without conceding that being accompanied is intrinsic-simply by insisting that being accompanied is syntactically equivalent to a d-relational property since it, itself, is d-relational.

27 As Hoffmann-Kolss puts it, "Francescotti does not specify a theory of identity of events which excludes a trivialization of his criterion. For suppose that $P$ is an arbitrary property of $x$ 's, that $R$ is the relation in which $x$ stands to $y$ iff $x$ has $P$ and $y$ has exactly the same qualitative properties as $x$ and that $\mathbf{C}$ consists of all individuals that have the same qualitative properties as $x$. Then in the same vein as one can argue that being taller than every red-headed person in the world is a universal d-relational property of $x$ 's, because $x$ 's having it is the same event as $x$ 's standing in the taller than relation to all red-headed persons in the world, one can argue that $x$ 's instantiating $P$ consists in $x$ 's standing in $R$ to all members of $\mathbf{C}$, viz. to all individuals having the same qualitative properties as $x "(2010$, pp. 91-2). 
It is true that for all I say about event/state-identity, (iii) is true. There might be good reason to reject (iii), but even so, it seems that the falsity of (iii) does not follow from the notion of intrinsicality itself. The notion of an intrinsic property is compatible with a variety of different views about the identity of events or states, and it seems compatible even with ones that entail (iii). It is true that given (iii), $\mathrm{R}_{\mathrm{I}}{ }^{\star}$ yields the result that there are no intrinsic properties and no properties had intrinsically. Yet, while this result certainly seems false, it is not clear that a definition of 'intrinsic' should entail that it is false, since the presence of intrinsicality, while presumably true, does not seem to be a conceptual truth. It is arguable, then, that as a definition of 'intrinsic' $R^{\star}{ }_{I}$ should not include an account of event- or state-identity that prevents (iii) from being true.

In fact, despite the silence regarding event/state-identity, what I did propose is probably not quite neutral enough. It's arguable that any definition of 'intrinsic' should remain silent on the nature of event-identity and the identity of states of affairs, facts, property-instances, occurrences, and episodes. A definition of intrinsicality should also remain neutral on the ontological status of, and even the existence of, any one of these types of entity. The nature of facts, events, states of affairs, and the rest are matters of great debate, and the notion of an intrinsic property does not in itself provide any answer to these controversial issues. To remain neutral on the nature of such items, we might adopt a suggestion made by Harris (2010). Rather than interpreting 'consists in' in $\mathrm{R}^{\star}$ above as talk about event- or state-identity, or as talk about the sameness of property-instances, or sameness of occurrences, or of facts, Harris proposes that we interpret 'consists in' in the relational account simply as 'the same as' or 'identical with' and leave it at that. That is why $\mathrm{R}_{\mathrm{I}}{ }^{\star}$ was phrased as claiming that x's having $\mathrm{F}$ is the same as x's having internal properties, $\mathrm{I}_{1}, \ldots, \mathrm{I}_{\mathrm{n}}$. How the true nature of the items that are the same is best construed is left entirely open.

d. A definition of intrinsicality should also remain neutral on whether properties are universals or tropes. However, if properties are universals, then they are distinct from the objects that exemplify them, and in that case for any property $\mathrm{F}$, $\mathrm{x}$ 's exemplifying $\mathrm{F}$ consists in a relation (the exemplification relation) that $\mathrm{x}$ bears to a distinct item (the universal, F-ness). So it seems that if properties are universals (which a definition of 'intrinsic' should allow), then with $\mathrm{R}_{\mathrm{I}}{ }^{\star}$ all properties are always had extrinsically.

One might attempt to avoid this problem by restricting the d-relations that yield extrinsicality to d-relations to particulars. Then F-ness being distinct from $\mathrm{x}$ would not be enough to make $\mathrm{F}$ an extrinsic feature of $\mathrm{x}$. However, this maneuver avoids the problem only by presupposing that properties are not particulars - a controversial claim which the notion of intrinsicality does not itself imply. So 
rather than restricting the d-relations that yield extrinsicality to d-relations to particulars, I suggested instead that "the question 'Is $\mathrm{F}$ an intrinsic property of $\mathrm{x}$ ?' should be interpreted as 'Does x's having $\mathrm{F}$ consist in a relation that $\mathrm{x}$ bears to a distinct item, other than F itself?'” (1999, p. 603). When we ask whether $\mathrm{F}$ is an intrinsic feature of an object, the answer our definition gives should not rely on any general view about the nature of properties (e.g., whether they are distinct from particulars) or how objects have them (e. g., by exemplifying universals or by containing tropes). There is good reason, then, to view the d-relations definitive of extrinsicality as relations other than mere exemplification.

However, Harris mentions a different and difficult problem that remains. “Abstract particulars are no less problematic than universals: no measurement would be intrinsic if a relation to an abstract particular were to qualify as a drelation" (Harris 2010, p. 471). Consider being 4 meters wide or having a mass of 1 kilo. These properties seem to be intrinsic. But the worry is that, assuming numbers exist, these properties count as extrinsic according to $\mathrm{R}_{\mathrm{I}}{ }^{*}$ (and other versions of $\mathrm{R}$ ) since an object's having them is a matter of its being related to distinct particulars.

To solve this "measurement" problem, Harris recommends restricting the drelations that yield extrinsicality to those one bears to concrete items. That way we avoid having to say that x's relation to the number 4 makes being 4 meters wide extrinsic. (The exemplification problem is also solved with Harris' restriction since the property $\mathrm{F}$ to which $\mathrm{x}$ relates is not concrete-either because properties are universals or because they are tropes, which are commonly viewed as abstract particulars.)

Restricting the d-relations that make properties extrinsic to those one bears to concrete items certainly is an attractive way to avoid the measurement problem. Unfortunately, with this restriction we face another difficulty. In some cases, a d-relation to an abstract item appears to be extrinsic. The property of coexisting with the number 9 seems extrinsic, and so does the property of being such that sets exist. ${ }^{28}$

But without a restriction on the d-relations that yield extrinsic properties, how can an advocate of the relational approach solve the measurement problem? We use the predicate 'is 4 meters wide' to denote the property of being 4 meters wide, but we might use the more cumbersome 'has a meter width measurement equal to 4 'instead. Doesn't the availability of this paraphrase make it clear that an object x's being 4 meters wide is the same as its having a meter

28 That's why Harris, as mentioned in section 1, attempts to defend the counter-intuitive claim that these properties are intrinsic. 
width measurement equal to 4? I don't think so. It seems that one can consistently deny that numbers exist while also accepting the truth of various measurement claims; anti-realism about numbers does not commit one to denying that objects have a certain width, length, weight, and other measurable features. So, it seems, it can be true that some object $\mathrm{x}$ is 4 meters wide even if there are no numbers. And if there were no numbers, then

(i) x's being 4 meters wide

would not consist in

(ii) X's bearing the having a meter width measurement equal to relation to the number 4.

But suppose that numbers do exist. Wouldn't it then be that (i) consists in (ii)? On many plausible construals of 'consists in', it would be true that (i) consists in (ii). One might even find it appropriate to say that (i) is grounded in (ii). However, even if (i) consists in (ii), in some strong sense of the phrase, there is still a difference between (i) and (ii). (i) does not require the existence of numbers; as was mentioned, an anti-realist about numbers need not deny the truth of measurement claims. But (ii) does require the existence of numbers; being related to the number 4 obviously requires the existence of the number 4. So what's required for (i) is not the same as what's required for (ii). It is arguable, then, that (i) is not identical with (ii) even if numbers do exist. And if this is right, then even though (ii) is identical with x's instantiation of a d-relational property, we can plausibly deny that this is true of (i). So with the emphasis on identity in $\mathrm{R}_{\mathrm{I}}^{\star}$ (in place of 'consists in' in $\mathrm{R}^{\star}$ or the appeal to grounding in $\mathrm{R}_{\mathrm{G}}$ ), it seems we can avoid the result that being 4 meters wide is an extrinsic feature of $\mathrm{x}$. So even if we allow that d-relations to abstract particulars yield extrinsicality, there is a plausible reason available, for proponents of $\mathrm{R}_{\mathrm{I}}{ }^{\star}$, to deny that all measurement claims describe the instantiation of extrinsic properties. (But note: it does not seem wise to add to our definition of 'intrinsic' a clause guaranteeing that at least some measurement claims describe intrinsic properties. A definition of 'intrinsic' should not itself entail that this is true; the definition in itself should not guarantee that any properties are had intrinsically.)

\section{Concluding Remarks}

We have seen that INPs are problematic for accounts of intrinsicality that do not hyperintensionally distinguish the intrinsic from the extrinsic. Another type of property that is known to be problematic for many of these same accounts is 
the non-qualitative ("impure") variety. A duplication based account, for instance, implausibly classifies haecceitistic identity properties (properties of the form, is identical with $x$ ) as extrinsic since these properties are not shared by any duplicate of an object other than the object itself. With Langton and Lewis (1998, p. 335), one might avoid the result that these identity properties are extrinsic by restricting the duplication account to qualitative properties (pure rather than impure properties)-and perhaps defending this restriction by arguing that the word 'intrinsic' is ambiguous between the qualitative sense and the non-qualitative sense. ${ }^{29}$ However, against the idea that there is a distinct qualitative notion of intrinsicality, Eddon claims that the qualitative notion merely picks out a proper subset of the set of intrinsic properties: "an 'intrinsic qualitative property' is simply an intrinsic property that is also qualitative” (2011, p. 323, fn. 22). This seems exactly right. It is most plausible to think that qualitative intrinsic properties comprise merely a subset of the class of intrinsic properties, and that they are intrinsic in the very same sense (whatever that might be) that non-qualitative intrinsic properties are intrinsic. A relational account nicely accommodates this intuition. On a relational account, qualitative intrinsic properties are intrinsic in the very same sense as the non-qualitative ones, by being instantiated in virtue of having internal/non-d-relational properties. The difference between the two groups is simply whether those internal properties are qualitative.

Recall the quote from Lewis at the start of this essay. One intuitive characterization of intrinsicality he mentions is the idea that a sentence or statement ascribing an intrinsic property to something is entirely about the thing itself. There is also the quite similar (and arguably the same) idea that a thing has its intrinsic properties in virtue of the way that thing itself and nothing else is, the intrinsic properties of something depending only on the thing itself. ${ }^{30}$ Relational approach $\mathrm{R}$ (and $\mathrm{R}_{\mathrm{I}}^{\star}$ in particular) clearly captures these aspects of the notion of intrinsicality; if x's having $\mathrm{F}$ is a matter of its internal, non-d-relational proper-

29 Sider contends that there is a qualitative sense of 'intrinsic' and this is the sense which duplication accounts are meant to capture. "As I see it, we have a notion of the qualitative intrinsic properties, which are had in virtue of the way objects are" (1996, p. 4). Vallentyne also identifies a qualitative sense of the word, which he calls the "narrow" sense. A property is intrinsic in the broad sense "just in case having it is appropriately independent of the existence of other objects," whereas a property is intrinsic in the narrow sense "just in case it is intrinsic in the broad sense and is a qualitative property" (1997, p. 215).

30 Although Weatherson and Marshall (2013, sec. 2) question whether the former "locality" construal is the same as the latter interpretation. 
ties, then and only then is x's having F solely a matter of what $\mathrm{x}$ itself is like, entirely about $\mathrm{x}$ itself, and not a matter of what the world distinct from $\mathrm{x}$ is like.

The duplication description, however, stands out as expressing something a bit different. As Lewis put it, "[i]f something has an intrinsic property, then so does any perfect duplicate of that thing" (1983a, p. 111). That's what G. E. Moore said about intrinsic value: if something possesses it to any degree, then "anything exactly like it, must, under all circumstances, possess it in exactly the same degree" (1922, p. 261). The duplication description does capture something crucial to the notion of an intrinsic property. If intrinsic properties are those something has "in itself," then it would seem that no matter what environmental differences there are, so long as two objects are duplicates, they will have all the same intrinsic properties. Of course, whether duplication tracks intrinsicality depends on the type of duplication. If we include the duplication of impure identity properties (of the form being identical with $x$ ), then necessarily for any $\mathrm{x}$, only $\mathrm{x}$ is a duplicate of $\mathrm{x}$, in which case, the duplication definition yields the implausible result that all of x's properties are intrinsic. On the other hand, if duplicates are supposed to be those that share all the same qualitative properties, then the problem is that being identical with $x$ is not classified as intrinsic. A relational approach helps make sense of what duplication has to do with intrinsicality. According to $\mathrm{R}_{\mathrm{I}}{ }^{\star}$, it is the duplication of one's internal properties that ensures the duplication of one's intrinsic properties and that is because the duplication of internal properties is what preserves those features of an object that are not had in virtue of what the rest of the world is like.

$\mathrm{R}_{\mathrm{I}}^{\star}$ nicely captures the common intuitive descriptions of intrinsicality in the quote from Lewis. $\mathrm{R}_{\mathrm{I}}^{\star}$ also handles well-known objections to other accounts of intrinsicality. Unlike the famous account of Langton and Lewis, $\mathrm{R}_{\mathrm{I}}{ }^{\star}$ can correctly classify disjunctive properties without the contentious notion of naturalness, it correctly classifies properties of the form being such that there is an $F$, and it correctly classifies border-sensitive properties, such as being a rock. ${ }^{31}$ There are other ac-

31 The first of these three objections has be raised by many, including Yablo (1999), Marshall and Parsons (2001), and Witmer et al. (2005). The second is presented by Marshall and Parsons (2001). And Sider (2001) raises the objection regarding border-sensitive properties. It should not be hard to see how $R_{I}{ }^{\star}$, with its hyperintensionality, allows that some disjunctive properties can be had intrinsically and also had extrinsically. Being the only $F$ is correctly classified as extrinsic by $R_{I}^{\star}$ since x's being the only $F$ is, in part, $x$ 's having the negative d-relational property of there not being any distinct Fs ("in part" given that x's being the only F requires $x$ not containing any Fs as proper parts and also implies that $\mathrm{x}$ is $\mathrm{F}$ ). And given that being a rock requires not being enclosed within a larger rock, x's being a rock is also (in part) the possession of a negative drelational property. 
counts that avoid these objections, ${ }^{32}$ but unlike $\mathrm{R}_{\mathrm{I}}{ }^{\star}$ many of these fail to accommodate the apparent hyperintensionality of the intrinsic/extrinsic distinction and also incorrectly classify non-qualitative properties. Like $\mathrm{R}_{\mathrm{I}}^{\star}$, a grounding account, such as Rosen's (2010) and Witmer's (2014), avoids each of these problems. However, as shown in section 3 (with great help from Marshall's 2013 discussion), it seems that intrinsic properties might be grounded in facts about distinct objects. As was also mentioned in section 3, it is arguable that a definition of 'intrinsic' should remain neutral on whether grounding talk describes a phenomenon that actually obtains. For these reasons, $\mathrm{R}_{\mathrm{I}}{ }^{\star}$ is preferable.

32 Weatherson's (2001) combinational view, for example, was explicitly introduced as a way to avoid these three objections. 


\section{References}

Bader, Ralf M. (2013). "Towards a Hyperintensional Theory of Intrinsicality.” The Journal of Philosophy 110(10): pp. 525-563.

Correia, Fabrice (2010). “Grounding and Truth-Functions.” Logique et Analyse 53 (211): pp. 251-279.

Dunn, J. Michael (1990). "Relevant Predication 2: Intrinsic Properties and Internal Relations." Philosophical Studies 60(3): pp. 177-206.

Eddon, Maya (2011). "Intrinsicality and Hyperintensionality." Philosophy and Phenomenological Research 82(2): pp. 314-336.

Figdor, Carrie (2008). “Intrinsically/Extrinsically.” The Journal of Philosophy 105(11): pp. $691-718$.

Figdor, Carrie (2014). “What's the Use of an Intrinsic Property?” In R. Francescotti (ed.), Companion to Intrinsic Properties. Berlin: De Gruyter.

Francescotti, Robert (1999). "How to Define Intrinsic Properties.” Noûs 33(4): pp. 590-609. Harris, Roger (2010). "How to Define Extrinsic Properties." Axiomathes 20(4): pp. 461-478. Hoffmann-Kolss, Vera (2010). The Metaphysics of Extrinsic Properties. Frankfurt: Ontos-Verlag. Hoffmann-Kolss, Vera (2014). "Is the Intrinsic/Extrinsic Distinction Hyperintentional?" In R. Francescotti (ed.), Companion to Intrinsic Properties. Berlin: De Gruyter.

Humberstone, I. Lloyd (1996). "Intrinsic/Extrinsic." Synthese 108(2): pp. 205-267.

Khamara, E. J. (1988). "Indiscernibles and the Absolute Theory of Space and Time." Studia Leibnitiana 20(2): pp. 140-159.

Langton, Rae and David Lewis. (1998). “Defining 'Intrinsic'." Philosophy and Phenomenological Research 58(2): pp. 333-345.

Lewis, David (1983a). “Extrinsic Properties.” Philosophical Studies 44(2): pp. 197-200.

Lewis, David (1983b). "New Work for a Theory of Universals.” Australasian Journal of Philosophy 61(4): pp. 343-377.

Lewis, David (1986). On the Plurality of Worlds. Oxford: Blackwell.

Marshall, Dan (2013). "Intrinsicality and Grounding." Philosophy and Phenomenological Research. Early view: published online, 3 July 2103.

Marshall, D. and J. Parsons (2001). "Langton and Lewis on 'Intrinsic'." Philosophy and Phenomenological Research 63(2): pp. 347-351.

Moore, G. E. (1922). Philosophical Studies. London: Routledge and Kegan Paul.

Rosen, Gideon (2010). “Metaphysical Dependence: Grounding and Reduction.” In B. Hale and A. Hoffmann (eds.), Modality: Metaphysics, Logic, and Epistemology (pp. 109-136). Oxford: Oxford University Press.

Schaffer, Jonathan (2012). "Grounding, Transitivity, and Contrastivity." In F. Correia and B. Schnieder (eds.), Metaphysical Grounding: Understanding the Structure of Reality (pp. 122-138). Cambridge: Cambridge University Press.

Sider, Theodore (1996). “Intrinsic Properties.” Philosophical Studies 83(1): pp. 1-27.

Sider, Theodore (2001). "Maximality and Intrinsic Properties." Philosophy and Phenomenological Research 63(2): pp. 357-364

Skiles, Alexander (2009). "Trogdon on Monism and Intrinsicality." Australasian Journal of Philosophy 87(1): pp. 149-154.

Trogdon, Kelly (2009). “Monism and Instrinsicality.” Australasian Journal of Philosophy 87(1): pp. $127-148$. 
Trogdon, Kelly (2010). “Intrinsicality for Monists (and Pluralists).” Australasian Journal of Philosophy 88(3): pp. 555-558.

Vallentyne, Peter (1997). “Intrinsic Properties Defined." Philosophical Studies 88(2): pp. 209-219.

Weatherson, Brian (2001). "Intrinsic Properties and Combinatorial Principles." Philosophy and Phenomenological Research 63(2): pp. 365-380.

Weatherson, Brian and Dan Marshall (2013). “Intrinsic vs. Extrinsic Properties.” In E. N. Zalta (ed.), The Stanford Encyclopedia of Philosophy (Spring 2013 edition). http://plato.stanford.edu/archives/spr2013/entries/intrinsic-extrinsic/

Witmer, D. Gene (2014). “A Simple Theory of Intrinsicality.” In R. Francescotti (ed.), Companion to Intrinsic Properties. Berlin: De Gruyter.

Witmer, D. Gene, Willaim Butchard, and Kelly Trogdon (2005). "Intrinsicality without Naturalness." Philosophical and Phenomenological Research 70(2): pp. 326-350. Yablo, Stephen (1999). “Intrinsicness.” Philosophical Topics 26(1-2): pp. 479-505. 